\title{
Results of a pilot study to provide evidence on the efficacy of vitamin B12 to normalise elevated homocysteine of vegetarians
}

\author{
D. Obersby, D.C Chappell, A. Dunnett and A.A Tsiami \\ School of Psychology, Social Work and Human Sciences, University of West London, London, TW8 9GA, UK
}

Homocysteine can be remethylated through the major folic cycle path. It is recycled to methionine in a reaction catalyzed by the enzyme methionine synthase and is absolutely dependent on the essential vitamin B12 methylcobalamin (MeCbl). All other forms of vitamin B12 must be converted to MeCbl prior to their usage ${ }^{(1)}$. This can prove to be problematic, particularly for the aged $^{(2,3)}$. Vegetarians are known to be susceptible to vitamin B12 deficiency, due to a lack of dietary animal produce, which can elevate plasma total homocysteine (tHcy). There is positive evidence that links elevated plasma (tHcy) with cardiovascular disease $(\mathrm{CVD})^{(4)}$.

The specific objective of the present study was to establish if supplementary MeCbl will optimise elevated levels of plasma tHcy of vitamin B12 deficient vegetarians.

The design of the study was a parallel double blind randomised placebo controlled trial. The treatment regimes were either a $1 \mathrm{mg}$ $\mathrm{MeCbl}$ lozenge (the intervention group) taken every second day for 16 weeks or identical placebo. Successful participants firstly completed a confidential questionnaire/application form and met the study criteria, which included having a baseline plasma tHcy level $>10 \mu \mathrm{molL}^{-1}$ and who had been practicing vegetarianism for $\geqslant 3$ years, age 18 to 65 years old were recruited via the Vegetarian and Vegan Societies. The subjects comprised lactovegetarians/ lactoovovegetarians $(n=25)$ and vegans $(n=14)$. During the study participant diets were closely supervised by the compilation of diet diaries. Homocysteine assay was conducted by the accredited and validated self-test kit supplied and tested by Yorktest Laboratories Ltd. The study was approved by the University Ethics Committee and conducted in accordance with the WMA declaration of Helsinki. The protocol for the study was registered with ClinicalTrials.gov Protocol Registration System. A paired samples t test on baseline and post intervention levels of plasma t Hcy was performed employing SPSS software, with the null hypothesis (H0) being defined as 'there will be no reduction in plasma tHcy following treatment' $\mathrm{H} 0$ was rejected if the change in plasma tHcy was not zero, but accepted if it was zero. The results are summarised below:

\begin{tabular}{|c|c|c|c|c|c|}
\hline $\begin{array}{l}\text { Lozenge type } \\
(\alpha=0.05)\end{array}$ & $\mathrm{n}$ & df & $P$ (2-tailed) & $\begin{array}{l}\text { Mean difference } \\
\left(\mu \mathrm{molL}^{-1}\right)\end{array}$ & $\begin{array}{l}95 \% \text { confidence interval } \\
\left(\mu \mathrm{molL}^{-1}\right)\end{array}$ \\
\hline Methylcobalamin & 20 & 19 & 0.00 & -7.1 & $(-9.35 .-4.85)$ \\
\hline Placebo & 19 & 18 & 0.16 & -1.1 & $(-2.70,0.48)$ \\
\hline
\end{tabular}

Test value $=0$

The results indicates that $\mathrm{H} 0$ can be rejected for the $\mathrm{MeCbl}$ group since $P<0.05$ and $\mathrm{CI} 95 \%$ does not pass through zero whereas the placebo group $\mathrm{H} 0$ is accepted, since $P>0.05$ and CI $95 \%$ passes through zero. The paired samples t test also demonstrated that $\mathrm{MeCbl}$ effectively optimised homocysteine levels of baseline mean value of $15.5 \mu \mathrm{molL} \mathrm{L}^{-1}$ to post intervention mean level of $8.4 \mu \mathrm{molL}^{-1}$ (not shown).

Examination of participant food diaries confirmed that strict vegetarian diets were maintained throughout the period of the study and that all other bio-regulation of homocysteine nutrients met RDA and AI values. Overall, it is therefore reasonable to deduce that the results of the study indicate that $\mathrm{MeCbl}$ optimises elevated levels of homocysteine (to $<10 \mu \mathrm{molL}^{-1}$ ) of vitamin $\mathrm{B} 12 \mathrm{deficient}$ vegetarians and will potentially reduce the risk of CVD together with providing data to perform a (probable first) definitive clinical trial.

These findings will also potentially inform the dietary advice given to vegetarians.

1. Herrmann W, Obeid R, Schorr H et al. (2005) Curr Drug Met 6, 47-53.

2. Pezacka E, Green R \& Jacobsen DW. (1990) Arch Biochem Biophys 2, 443-450.

3. Kelly G. (1997) Alt Med Rev 2, 459-471.

4. Wald DS, Law M \& Morris JK. (2002) Brit Med J 325, 1202. 